\title{
Thermoelectric effects in graphene with local spin-orbit interaction
}

\author{
M. I. Alomar and David Sánchez \\ Institut de Física Interdisciplinària i Sistemes Complexos IFISC (UIB-CSIC), E-07122 Palma de Mallorca, Spain \\ and Departament de Física, Universitat de les Illes Balears, E-07122 Palma de Mallorca, Spain \\ (Received 14 January 2014; revised manuscript received 26 February 2014; published 17 March 2014)
}

\begin{abstract}
We investigate the transport properties of a graphene layer in the presence of Rashba spin-orbit interaction. Quite generally, spin-orbit interactions induce spin splittings and modifications of the graphene band structure. We calculate within the scattering approach the linear electric and thermoelectric responses of a clean sample when the Rashba coupling is localized around a finite region. We find that the thermoelectric conductance, unlike its electric counterpart, is quite sensitive to external modulations of the Fermi energy. Therefore, our results suggest that thermocurrent measurements may serve as a useful tool to detect nonhomogeneous spin-orbit interactions present in a graphene-based device. Furthermore, we find that the junction thermopower is largely dominated by an intrinsic term independently of the spin-orbit potential scattering. We discuss the possibility of canceling the intrinsic thermopower by resolving the Seebeck coefficient in the subband space. This causes unbalanced populations of electronic modes which can be tuned with external gate voltages or applied temperature biases.
\end{abstract}

DOI: 10.1103/PhysRevB.89.115422

PACS number(s): 72.80.Vp, 73.22.Pr, 71.70.Ej, 72.20.Pa

\section{INTRODUCTION}

Graphene is a single layer of carbon atoms arranged on a two-dimensional honeycomb lattice [1,2]. The study of its electronic properties has recently attracted great interest [3-6] in part due to peculiar features of its energy band structure. Within a tight-binding model, graphene's conduction and valence bands touch each other at six different points, the $K$ points, which reduce to two, $K$ and $K^{\prime}$, because the rest are equivalent by symmetry. Near these points and at low energies, electrons behave as massless fermions traveling at fixed velocity $V_{F} \sim 10^{6} \mathrm{~m} / \mathrm{s}$, independently of their energy. Then, the energy spectrum consists of two cones that come into contact at their vertices and the low-energy excitations can be conveniently described by an effective Dirac-Weyl equation where the speed of light is replaced with $V_{F}$ [3].

Recent works suggest large spin-orbit strengths in graphene layers under the influence of metallic substrates [7-12]. This finding is interesting in view of recent studies that relate spin-orbit coupling of the Rashba type $[13,14]$ to topological insulating behavior $[15,16]$. Importantly, the Rashba coupling strength can be externally tuned by modifying the electric field applied to a nearby gate [17]. This type of interaction leads to band splittings and enriched spintronic effects $[18,19]$. In semiconductor quantum wires with parabolic confinement, the presence of localized Rashba interaction has been predicted to yield Fano antiresonances [20-25], to help the detection of entangled electrons [26-28], and to assist electron-spin resonance manipulation [29-31]. The effect of nonhomogeneous Rashba couplings has also been considered in the transport characteristics of two-dimensional systems [32-36]. A natural question is thus to what extent these results are modified in graphene monolayers. In contrast to semiconductor heterojunctions, in graphene electrons are massless and the spin-orbit interaction depends on a pseudospin degree, not a momentum.

In this paper, we investigate the influence of local Rashba spin-orbit interaction on the electric and thermoelectric properties of graphene. We shall focus on the linear regime of transport. Previous studies have considered Fano line shapes in graphene junctions [37,38], spin densities in nanoribbons [39] and superlattices [40], spin-dependent transmissions [41], and Klein (chiral) tunneling [42]. Here, we are mainly concerned with the voltages generated in response to a temperature difference (the Seebeck effect) [43]. Interestingly, recent results indicate enhanced thermopower in graphene monolayers [44-46], which paves the way for promising applications to achieve efficient heat-to-energy converters [47]. Here we discuss the possibility of manipulating the thermopower with a local spin-orbit interaction. In fact, we find that a spin-orbit graphene monolayer is more sensitive to temperature biases than to voltage differences. Furthermore, since the Rashba coupling splits the graphene electronic band structure (see Fig. 1), the transmission thus depends on the subband index. In analogy with spin caloritronic devices [48], where a thermal gradient induces a spin-polarized voltage bias [49,50], we propose to use the Seebeck effect to generate a difference between occupations with different subband indices.

The paper is organized as follows. In Sec. II, we describe our model Hamiltonian and investigate the effects of a uniform spin-orbit coupling in both the energy spectrum and the electronic states of a flat graphene sheet. Section III describes the system under consideration: a junction with a Rashba interaction potential localized around a central region where the spin-orbit strength is nonzero and constant. Using matching methods for wave functions with four components, we find the transmission probabilities for all incident electronic modes. Importantly, the transmission function shows, for a given subband index, a critical angle beyond which electrons cannot be transmitted across the junction. The electric conductance and the subband polarization are discussed in Sec. IV. We find that the polarization rapidly changes in the energy scale of the Rashba strength for sufficiently wide spin-orbit regions. Section V contains the central results of our work. We calculate the thermocurrent in response to a small temperature shift and obtain strong modulations when the Fermi energy is tuned even to values much larger than the spin-orbit strength. Surprisingly, the Seebeck coefficient is a smooth function of energy, an effect which we attribute to a background intrinsic thermopower which is dominant for a wide range of Fermi energies. We then 


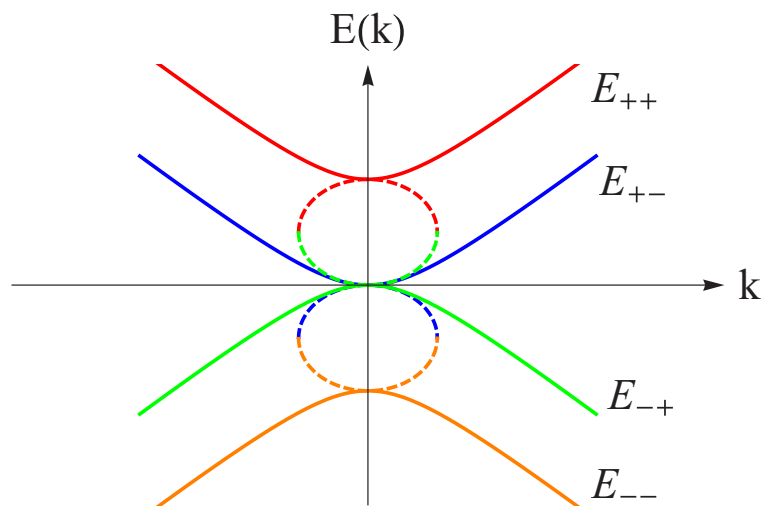

FIG. 1. (Color online) Sketch of the energy band structure of a graphene layer with spin-orbit interaction of the Rashba type. Solid lines indicate propagant states, while dashed lines depict the energy associated to evanescent states. Due to the spin-orbit potential, four bands (labeled as,,+++--+ , and -- ) are obtained.

determine the subband thermovoltage generated in response to a temperature bias and recover the strong variation with energy, yielding positive or negative population imbalances depending on the value of the externally tuned Fermi energy. Finally, our conclusions are summarized in Sec. VI.

\section{THEORETICAL MODEL}

We consider a graphene layer in the $x y$ plane with spatially varying spin-orbit interaction along the $x$ direction. Within the continuum limit, the total Hamiltonian reads

$$
\begin{aligned}
\mathcal{H}= & -i \hbar V_{F}\left(\sigma_{x} \partial / \partial x+\sigma_{y} \partial / \partial y\right) \otimes s_{o} \\
& +\lambda\left(\sigma_{x} \otimes s_{y}-\sigma_{y} \otimes s_{x}\right) .
\end{aligned}
$$

The first term in the right-hand side represents the effective Hamiltonian for electrons in a clean graphene sheet. This model is valid when intervalley scattering can be safely neglected. The electron spin and pseudospin (sublattice) degrees of freedom are taken into account with the Pauli $s$ and $\sigma$ matrices, respectively. The second term describes the Rashba spin-orbit coupling, with $\lambda$ the interaction strength [15]. We take $\lambda$ as a slowly varying function in a length scale larger than the graphene lattice constant. Thus, the continuum model remains valid. Furthermore, in Eq. (1), we have neglected the intrinsic contribution to the spin-orbit interaction since this term is much smaller than the Rashba coupling and cannot be externally tuned $[51,52]$.

Let $k(q)$ be the wave-vector component along the $x(y)$ direction. Then, the eigenenergies of $\mathcal{H}$ are given by

$$
E_{l, n}=l \sqrt{\lambda^{2}+\hbar^{2} V_{F}^{2}\left(k^{2}+q^{2}\right)}+n \lambda,
$$

where $l= \pm$ labels states with positive or negative energies and $n= \pm$ is the subband index. The energy spectrum is plotted with solid lines in Fig. 1 for a finite value of the spin-orbit strength $\lambda$. The energy bands split with a splitting given by $2 \lambda$ for both the positive and negative branches of the spectrum.

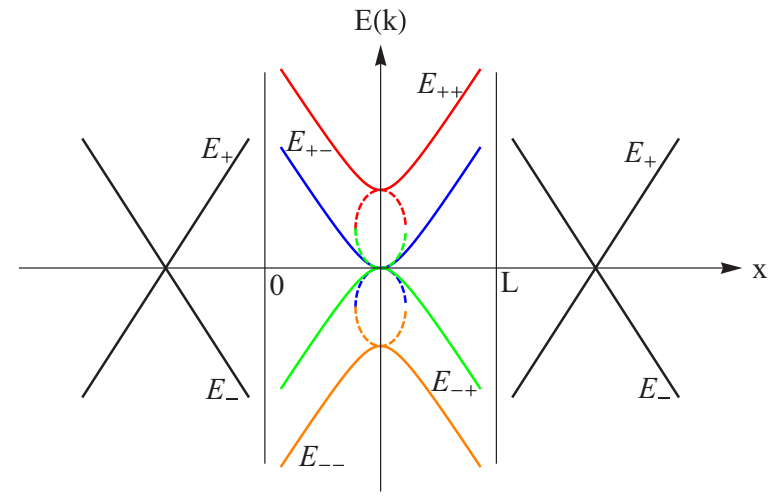

FIG. 2. (Color online) Pictorial representation of a graphene layer with a central region of length $L$ where spin-orbit interaction is active. We take $x$ as the propagation direction. We show the energy spectra both inside and outside the central region.

The eigenstates of $\mathcal{H}$ are

$\psi_{l, n}^{m}(x)=\frac{e^{i m k x} e^{i q y} / 2}{\sqrt{\hbar^{2} V_{F}^{2}\left(k^{2}+q^{2}\right)+E_{l, n}^{2}}}\left(\begin{array}{c}-i n \hbar V_{F}(m k-i q) \\ E_{l, n} \\ -i n E_{l, n} \\ \hbar V_{F}(m k+i q)\end{array}\right)$,

where we explicitly indicate the propagation direction with the aid of the index $m= \pm$, which determines the sign of the momentum along $x$. Since the scattering potential is invariant in the $y$ direction, we take $q$ as a real quantity. However, the $k$ momentum can be real or purely imaginary depending on whether one deals with traveling or evanescent waves. A systematic method of finding evanescent states in quantum wires with Rashba interaction is presented in Ref. [53]. Here, we notice that the energy of evanescent waves emerges from the subband spectra and coalesces for $E= \pm \lambda$ (see the dashed lines in Fig. 1).

In the absence of spin-orbit interaction, we recover the well-known dispersion relation for bare graphene,

$$
E_{l}=l \hbar V_{F} \sqrt{k^{2}+q^{2}},
$$

with eigenstates

$$
\psi_{l, n}^{m}(x, y)=\frac{e^{i m k x} e^{i q y}}{2}\left(\begin{array}{c}
-i n m e^{-i m \phi} \\
l \\
-i n l \\
m e^{i m \phi}
\end{array}\right)
$$

Here, $\phi$ is the wave-vector angle defined as $\phi=\tan ^{-1} q / k$. We represent Eq. (4) for $q=0$ in the left and right sides of Fig. 2. The spectrum $E(k)$ is linear with a constant slope. In contrast, in the presence of Rashba interaction, the energy bands become parabolic for energies small compared to the spin-orbit strength (central area in Fig. 2).

\section{LOCAL RASHBA INTERACTION}

We investigate the scattering problem sketched in Fig. 2 with three distinct regions. While the side regions (left and right) are bare graphene, the central region of length $L$ is subjected to spin-orbit interaction of the Rashba type. Since 
the problem is invariant in the direction perpendicular to $x$, the $y$ component of the momentum does not change and we can write it in terms of the wave-vector angle,

$$
q=\frac{E}{\hbar V_{F}} \sin \phi
$$

We consider electrons with fixed energy $E>0$. From Eqs. (2) and (6), we obtain the wave-vector component parallel to the transport direction,

$$
k=E \sqrt{\left(1-\sin ^{2} \phi\right)} / \hbar V_{F},
$$

valid for $x<0$ and $x>L$. For $0<x<L, k$ can be determined from Eqs. (2) and (4):

$$
k^{n}=\sqrt{E\left(E-2 n \lambda-E \sin ^{2} \phi\right)} / \hbar V_{F} .
$$

In the central region, we have two possible values for $k^{n}$, i.e., one for the subband with $n=+$ and one for the subband with $n=-$, although a more careful analysis is needed in terms of the subband index. First, we notice that, in general, for any energy, the momentum is always real if $E-2 n \lambda-E \sin ^{2}$ $\phi>0$, i.e.

$$
\sin \phi<\sqrt{\frac{E-2 n \lambda}{E}} .
$$

Now, for $E>2 \lambda$ and $n=-$, Eq. (9) is always satisfied since $\sin \phi$ is bounded between 0 and 1 . In contrast, for $n=+$, we have a critical angle at which the momentum becomes pure imaginary. For angles higher than the critical angle, we have an evanescent wave. Then, for $0<E<2 \lambda$ and $n=-$, Eq. (9) also holds as before, but for $n=+$ the momentum becomes pure imaginary since Eq. (9) is never satisfied and the wave is evanescent for any value of the angle $\phi$. Similar critical angles have been invoked to discuss total internal reflection effects in semiconductor interfaces with spin-orbit interaction [33].

We are now in a position to solve the scattering problem in Fig. 2. We focus on the case $E>0$ since our system exhibits particle-hole symmetry, even in the presence of Rashba coupling. Therefore, we take $l=+1$. We consider a most simple inhomogeneity, namely, $\lambda=0$ for $x<0$ and $x>L$, and $\lambda$ nonzero and uniform for $0<x<L$. This is not contradictory with the assumption that $\lambda$ is a slowly varying function in an atomic level, because the scale over which this change takes place is much bigger than the graphene lattice constant. The matching method allows us to calculate all reflection and transmission amplitudes for a given electron, which we take as impinging from the left. In the following, we express the wave function at each region as $\psi_{+, n}^{m}(x, y)=$ $\psi_{n}^{m} e^{i m k x} e^{i q y}$ [cf. Eq. (11)]. We first specify the left $(\ell)$ wave function for $x<0$ :

$$
\begin{aligned}
\psi_{\ell, n}(x, y)= & \psi_{n}^{+} e^{i k x} e^{i q y}+r_{n,-} \psi_{-}^{-} e^{-i k x} e^{i q y} \\
& +r_{n,+} \psi_{+}^{-} e^{-i k x} e^{i q y},
\end{aligned}
$$

where the incident subband $n$ can be taken as + or - . The reflection amplitudes $r_{n,-}$ and $r_{n,+}$ describe back scattering into - and + modes, respectively. Then, we have an incident wave with positive group velocity, $v=k>0$, and two reflected waves with $v=-k<0$, with the latter belonging to the doubly degenerate $E_{+}$branch in Fig. 2 .
In the central $(c)$ region, we have four coexisting waves,

$$
\begin{aligned}
\psi_{c, n}(x, y)= & a_{n,-} \psi_{-}^{+} e^{i k^{-} x} e^{i q y}+b_{n,+} \psi_{+}^{+} e^{i k^{+} x} e^{i q y} \\
& +c_{n,-} \psi_{-}^{-} e^{-i k^{-} x} e^{i q y}+d_{n,+} \psi_{+}^{-} e^{-i k^{+} x} e^{i q y},
\end{aligned}
$$

where the coefficients $a, b, c$, and $d$ are labeled with the incident subband $n$ and the wave-vector index \pm defined in Eq. (8). Note that the propagating or evanescent character of the partial waves is determined by the real or imaginary value of $k^{ \pm}$. Equation (11) is valid for $E>\lambda$, but for $0<E<\lambda$ we need to take into account the evanescent states taking $\psi_{+}^{+}$and $\psi_{+}^{-}$for $l=-1$.

Finally, in the right $(r)$ region, we only have transmitted waves with positive group velocity and positive and negative $n$ :

$$
\psi_{r, n}(x, y)=t_{n,-} \psi_{-}^{+} e^{i k x} e^{i q y}+t_{n,+} \psi_{+}^{+} e^{i k x} e^{i q y},
$$

where $t_{n, \pm}$ denotes the transmission amplitude from the $n$th incident subband toward the \pm mode.

At the boundaries $x=0$ and $x=L$, we impose continuity of the wave function,

$$
\begin{aligned}
& \psi_{\ell, n}(0, y)=\psi_{c, n}(0, y), \\
& \psi_{c, n}(L, y)=\psi_{r, n}(L, y),
\end{aligned}
$$

from which the eight coefficients $r_{n, \pm}, a_{n,-}, b_{n,+}, c_{n,-}, d_{n,+}$, and $t_{n, \pm}$ are determined.

In elastic scattering, the probability current is conserved. Since our system shows scattering along $x$ only, the current conservation condition leads to

$$
1+R_{n,+}+R_{n,-}=T_{n,+}+T_{n,-},
$$

where $R_{n, \pm}=\left|r_{n, \pm}\right|^{2}\left(T_{n, \pm}=\left|t_{n, \pm}\right|^{2}\right)$ is the reflection (transmission) probability. Due to the spin-chiral nature of the carriers [40], the off-diagonal probabilities $T_{+,-}$and $T_{-,+}$ vanish altogether and the spin-orbit interaction does not couple states with opposite subband indices. Figure 3 shows $T_{+,+}$ and $T_{-,-}$for $E>2 \lambda$ as a function of the incident angle. At low angles $(\phi \simeq 0)$, the transmission is close to unity. This

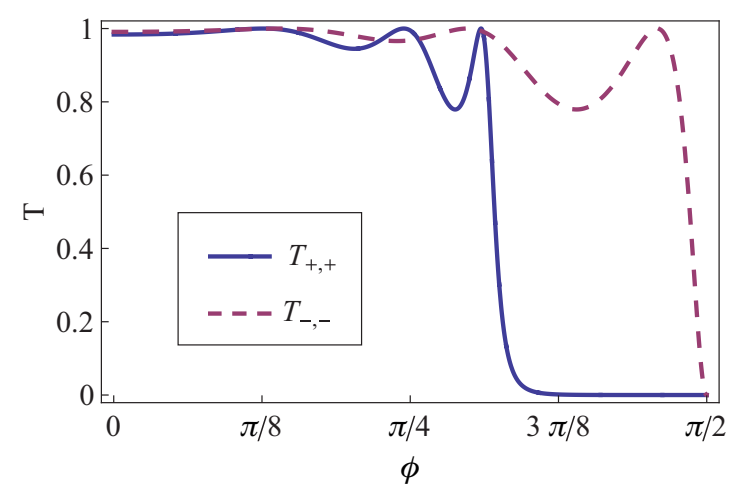

FIG. 3. (Color online) Transmission probability as a function of the incident angle $\phi$ for $E>2 \lambda$. The solid curve represents the transmission from $n=+$ to $n=+$ and the dashed curve the transmission from $n=-$ to $n=-$. Parameters: $\lambda=10 \mathrm{meV}$, $E=80 \mathrm{meV}$, and $L=100 \mathrm{~nm}$. 
is a manifestation of Klein tunneling in graphene for incident wave vectors parallel to the transport direction [54]. When $\phi$ rotates from 0 , the transmission departs from 1 due to scattering at the boundaries. The situation is akin to a single-barrier potential [54], but in our case the effect originates from a purely spin-orbit field.

Interestingly, in Fig. 3, we can see the emergence of a critical angle for $T_{+,+}$beyond which the transmission probability vanishes (solid line). It occurs because when we surpass the critical angle given by Eq. (9), the wave into the central region becomes evanescent and the transmission drops. The transition is not abrupt since there are tunneling contributions to $T_{+,+}$, but this effect is very weak. Note that $T_{-,-}$(dashed line) does not show any critical angle, as predicted by Eq. (9). Additionally, we also observe in Fig. 3 transmission resonances which we attribute to central waves interfering constructively for specific values of the incident angles.

\section{ELECTRIC CONDUCTANCE}

Within the scattering approach, the electric current carried by electrons in subband $n$ is obtained from the transmission probabilities integrated over the injecting energies $E$ and the wave-vector angle $\phi$,

$$
\begin{aligned}
I_{n}= & \frac{2 e W}{\pi h} \int_{0}^{\pi / 2} \cos \phi d \phi \int_{-\infty}^{\infty} \mathcal{K}(E) T_{n, n}(E, \phi) \\
& \times\left[f_{L}(E)-f_{R}(E)\right] d E,
\end{aligned}
$$

where $W$ is the sample width in the $y$ direction and $f_{\ell}(E)$ and $f_{r}(E)$ are Fermi-Dirac distribution functions that describe the electronic population both in the left and right side, asymptotically far from the scattering (central) region. The 2 factor is due to the valley degeneracy. In Eq. (16), $\mathcal{K}(E)=$ $E / \hbar V_{F}$ is obtained from the graphene dispersion relation, given by Eq. (4). The total current is thus $I=\sum_{n} I_{n}$.

To obtain the linear conductance $G=(d I / d V)_{V=0}$, a small voltage bias $V$ is applied across the junction. We can shift the left Fermi-Dirac distribution $f_{\ell}=f(E-e V)$ fixing the right one $f_{r}=f(E)$, where $f(E)=1 /\left(1+e^{\left(E-E_{F}\right) / k_{B} T_{0}}\right)$ is the equilibrium distribution function with $E_{F}$ the Fermi energy and $T_{0}$ the background temperature. After Taylor expanding Eq. (16) up to first order in $V$, we find at zero temperature $G=\sum_{n} G_{n}$, where

$$
G_{n}=G_{0} \int_{0}^{\pi / 2} T_{n, n}\left(E_{F}, \phi\right) \cos \phi d \phi,
$$

and $G_{0}=2 e^{2} W \mathcal{K}_{F} / \pi h=4 e^{2} W E_{F} / h^{2} V_{F}$ is the maximum conductance of an ideal two-dimensional conductor since $\operatorname{Int}\left(W \mathcal{K}_{F} / \pi\right)$ is the number of open channels of a sample with Fermi wave number $\mathcal{K}_{F}$ [55].

Figure 4 shows the conductance as a function of $E_{F}$. We choose the Fermi energy as the changing parameter since it can be easily tuned in an experimental setup [1]. The conductance $G_{+}$(blue, dashed line) is small for energies between 0 and $2 \lambda$. This is because in this energy range electrons from subband + can be transmitted only via a conventional tunneling effect due to the center energy splitting. The transmission probability is thus small. For $E_{F}$ higher than $2 \lambda, G_{+}$increases since traveling

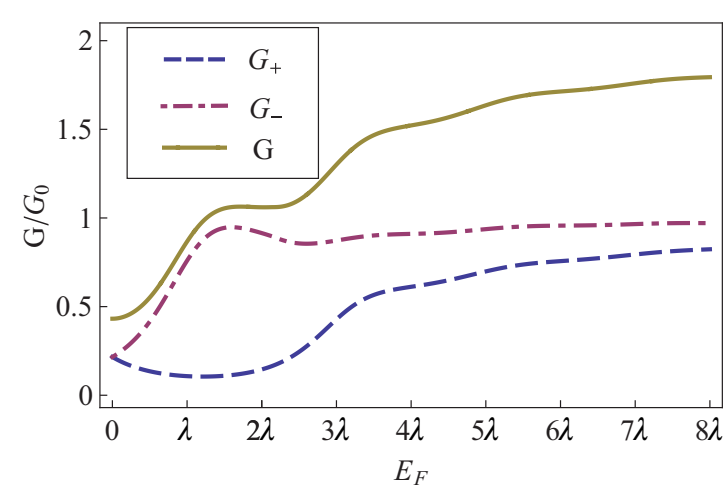

FIG. 4. (Color online) Conductance as a function of Fermi energy. Parameters: $\lambda=10 \mathrm{meV}$ and $L=100 \mathrm{~nm}$.

waves are now permitted in the central region. However, the increase is slow due to the persistence of the critical angle above which the transmission probability is zero. The conductance for the - subband $\left(G_{-}\right)$is always close to the maximal value $G_{0}$ because for this mode there always exists a traveling wave in the central region. In general, the total conductance $G=G_{+}+G_{-}$(solid line) is a monotonically increasing function of the Fermi energy and tends to $2 G_{0}$ at large energies where spin-orbit scattering is less efficient.

In Fig. 5, we represent the subband polarization defined as

$$
P=\frac{G_{+}-G_{-}}{G_{+}+G_{-}} .
$$

We can see that for $\lambda<E_{F}<2 \lambda$, most of the electrons have negative polarization because for those energies the wave with positive $n$ becomes evanescent inside the central region and the transmission probability is very small. This effect is more visible for wider regions of the spin-orbit stripe. As we increase the Fermi energy, there are more electrons with positive polarization since for $E_{F}>2 \lambda$ the states with $n=+$ are traveling waves and their transmission probability is larger. Clearly, in the limit $E_{F} \gg \lambda$, electron scattering is insensitive to the spin-orbit potential and the distinction between the + and - subbands vanishes, yielding $P \rightarrow 0$.

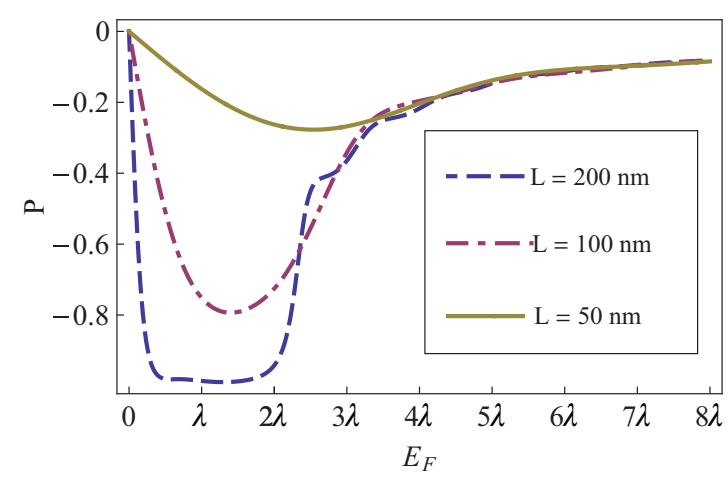

FIG. 5. (Color online) Subband polarization of the conductance as a function of Fermi energy for $\lambda=10 \mathrm{meV}$ and different values of the spin-orbit region length. 


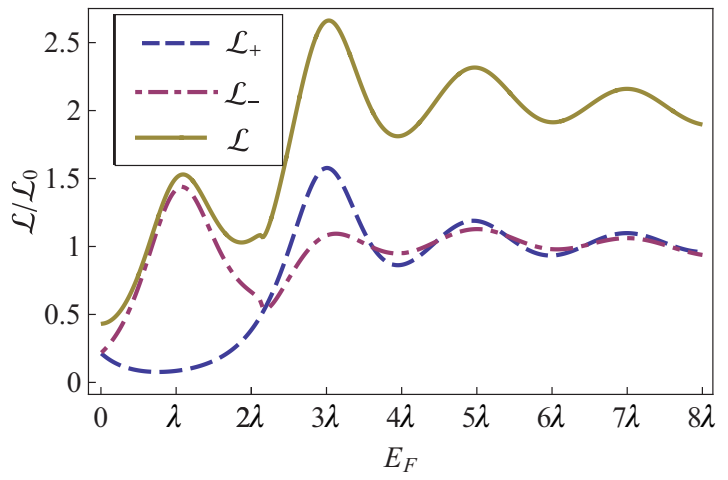

FIG. 6. (Color online) Thermoelectric conductance as a function of Fermi energy. Parameters: $\lambda=10 \mathrm{meV}$ and $L=100 \mathrm{~nm}$.

\section{THERMOELECTRIC CONDUCTANCE}

The current generated in the linear regime in response to a small temperature difference $\Delta T$ applied across the junction can be obtained from Eq. (16), replacing the left Fermi-Dirac distribution with $f\left(E, T_{0}+\Delta T\right)$ and the right one with $f\left(E, T_{0}\right)$ :

$$
\begin{aligned}
I_{n}= & \frac{2 e W}{h \pi} \frac{\Delta T}{T_{0}} \int_{0}^{\pi / 2} \cos \phi d \phi \int \frac{E}{\hbar V_{F}}\left(E-E_{F}\right) \\
& \times\left(-\frac{\partial f}{\partial E}\right) T_{n, n}(E, \phi) d E .
\end{aligned}
$$

We are interested in the low-temperature regime. Then, to leading order in a Sommerfeld expansion, the thermoelectric conductance reads $\mathcal{L}=I / \Delta T=\sum_{n} \mathcal{L}_{n}$, where

$$
\begin{aligned}
\mathcal{L}_{n}= & \sum_{n} \mathcal{L}_{0}\left[\int_{0}^{\pi / 2} T_{n, n}\left(E_{F}, \phi\right) \cos \phi d \phi\right. \\
& \left.+E_{F} \frac{\partial}{\partial E_{F}} \int_{0}^{\pi / 2} T_{n, n}\left(E_{F}, \phi\right) \cos \phi d \phi\right],
\end{aligned}
$$

where $\mathcal{L}_{0}=k_{B}^{2} e W T_{0} / 3 \hbar^{2} V_{F}$.

In Fig. 6, we represent the thermoelectric conductance as a function of the Fermi energy. Surprisingly, we observe strongly modulated oscillations with a decreasing amplitude as we increase $E_{F}$. This implies that the thermocurrent is more sensitive than the electric current to small variations of $E_{F}$. Furthermore, we find that the position difference between consecutive peaks in $\mathcal{L}$ is approximately given by the spin-orbit strength $\lambda$. Therefore, thermoelectric measurements can be rather useful in the detection of local spin-orbit fields in graphene single layers.

By virtue of the Seebeck effect, we expect that a thermovoltage will be generated when the junction is in the presence of a temperature gradient under open circuit conditions [43]. To keep our discussion general, we consider different electrochemical potentials $\mu_{\alpha n}=E_{F}+e V_{n}$ for each subband, where $\alpha=\ell, r$. Both sides of the junction are maintained at different temperatures, $T_{\alpha}$, independently of $n$. Then, the current flowing in the $n$ mode in response to small shifts $\mu_{\ell n}-\mu_{r n}$ and $T_{\ell}-T_{r}$ is

$$
I_{n}=\frac{\mu_{\ell n}-\mu_{r n}}{e} G_{n}+\left(T_{\ell}-T_{r}\right) \mathcal{L}_{n},
$$

where the transport coefficients $G_{n}$ and $\mathcal{L}_{n}$ are given by Eqs. (17) and (20), respectively.

We define [56]

$$
\begin{aligned}
\Delta T & =T_{l}-T_{r}, \\
\mu_{\alpha} & =\frac{1}{2}\left(\mu_{\alpha+}+\mu_{\alpha-}\right), \\
e V & =\mu_{\ell}-\mu_{r}, \\
e V_{s} & =\left(\mu_{\ell+}-\mu_{\ell-}\right)-\left(\mu_{r+}-\mu_{r-}\right),
\end{aligned}
$$

where $\Delta T$ is the temperature difference, $V$ is the bias voltage, and $V_{s}$ is the subband voltage that takes into account possible voltage differences in the same lead between different subbands [57]. Using Eq. (22) in Eqs. (16) and (19), we find the total current

$$
I=\left(G_{+}+G_{-}\right) V+\frac{1}{2}\left(G_{+}-G_{-}\right) V_{s}+\left(\mathcal{L}_{+}+\mathcal{L}_{-}\right) \Delta T,
$$

and the subband current $I_{s}=I_{+}-I_{-}$,

$$
I_{s}=\left(G_{+}-G_{-}\right) V+\frac{1}{2}\left(G_{+}+G_{-}\right) V_{s}+\left(\mathcal{L}_{+}-\mathcal{L}_{-}\right) \Delta T .
$$

We note that $I_{s}$ is a polarization current in the subband space. It then plays the role analogous to a spin or pseudospin current since $n$ can take on two values only.

In Eqs. (23) and (24), the transport coefficients are given by Eqs. (17) and (20). Defining the integrated transmission per subband as

$$
\mathcal{T}_{n}\left(E_{F}\right)=\int_{0}^{\pi / 2} T_{n, n}\left(E_{F}, \phi\right) \cos \phi d \phi,
$$

Eqs. (17) and (20) can be recast in the form

$$
\begin{aligned}
G_{n} & =\left(\frac{e}{\pi \hbar}\right)^{2} \frac{W E_{F}}{V_{F}} \mathcal{T}_{n}\left(E_{F}\right), \\
\mathcal{L}_{n} & =\frac{e k_{B}^{2}}{3 \hbar^{2}} \frac{W T_{0}}{V_{F}}\left[\mathcal{T}_{n}\left(E_{F}\right)+E_{F} \frac{\partial \mathcal{T}_{n}}{\partial E_{F}}\right],
\end{aligned}
$$

where $\partial \mathcal{T}_{n} / \partial E_{F}$ is the energy derivative of $\mathcal{T}_{n}$ evaluated at $E_{F}$.

Interestingly, the low-temperature conductance is given by the integrated transmission, in agreement with the Landauer picture of transport, but the thermoelectric conductance contains an additional term. This can be seen more clearly in the calculation of the charge thermopower or Seebeck coefficient $S=(V / \Delta T)_{I=0, I_{s}=0}$, which determines the voltage generated in the junction in response to a temperature shift when both the total and subband currents are set to zero. From Eqs. (23) and (24), we find

$$
S=-\frac{1}{2}\left(\frac{\mathcal{L}_{+}}{G_{+}}+\frac{\mathcal{L}_{-}}{G_{-}}\right) .
$$

Inserting Eqs. (26) in Eq. (27), we obtain the lowtemperature thermopower,

$$
S=-\frac{\pi^{2} k_{B}}{3 e} \frac{k_{B} T_{0}}{E_{F}}\left(1+E_{F} \frac{\sum_{n} \partial \mathcal{T}_{n} / \partial E_{F}}{\sum_{n} \mathcal{T}_{n}}\right) .
$$

We notice two contributions in Eq. (28). The second term in brackets can be understood with the aid of the Mott formula $S \propto \partial \ln G / \partial E_{F}$, which is expected to hold in generic 


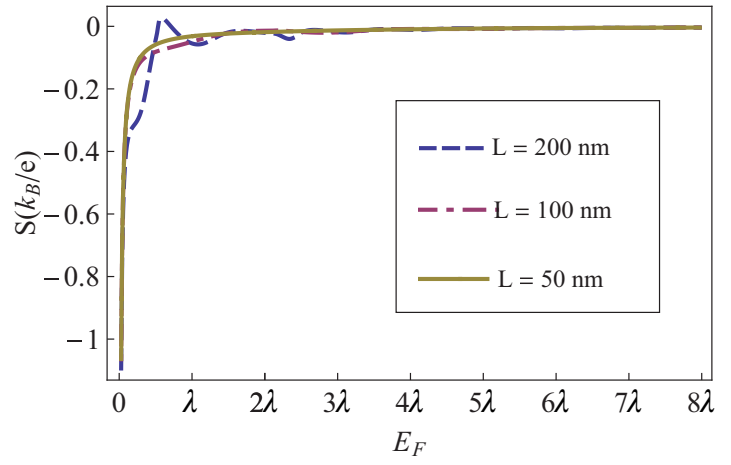

FIG. 7. (Color online) Seebeck coefficient as a function of Fermi energy for different values of the spin-orbit region length. Parameters: $\lambda=10 \mathrm{meV}$ and $T_{0}=1 \mathrm{~K}$.

conductors at low temperature. It is a single-particle result, which is satisfied in low-dimensional systems such as quantum dots [58] and quantum point contacts [59], for which a sizable thermopower is detected only if the transmission strongly depends on energy. It is thus a pure transport contribution. However, Eq. (28) shows an additional term which is insensitive to transmission modulations. In fact, for a constant transmission probability or when $\mathcal{T}_{n}$ shows a weak variation with energy on the scale of $E_{F}$, Eq. (28) reduces to

$$
S \simeq-\frac{\pi^{2} k_{B}}{3 e} \frac{k_{B} T_{0}}{E_{F}} .
$$

This intrinsic contribution is independent of the sample details and, more importantly, survives in the purely ballistic limit. It simply states that in the highly degenerate limit $\left(E_{F} \gg T_{0}\right.$, i.e., the range of validity of the Sommerfeld approximation), the thermopower is given by the entropy per unit charge $\left(k_{B} / e\right)$ associated to the fraction of the electron density which is thermally excited $\left(k_{B} T_{0} / E_{F}\right)$. Therefore, Eq. (29) is completely general and does not depend on the nature of the scattering potential. For $E_{F}=1 \mathrm{meV}$ and $T_{0}=1 \mathrm{~K}$, a typical value for the intrinsic thermopower yields $S=20 \mu \mathrm{V} / \mathrm{K}$, a value detectable with present techniques [45].

We thus expect a competition between the intrinsic and the transport terms in the Seebeck coefficient. We plot $S$ in Fig. 7 as a function of $E_{F}$ for a nonzero value of the Rashba strength. We observe that the junction thermopower is always negative, indicating that when the left side is hotter than the right side, the system generates a negative bias to compensate the excess of thermally activated electrons. Furthermore, $S$ is quite robust to variations of the spin-orbit region size $L$. Additionally, the overall shape of $S$ is rather smooth, unlike the strong variation of the thermoelectric conductance as $E_{F}$ increases (cf. Fig. 6). These facts can be explained taking into account the intrinsic thermopower written in Eq. (29). At high energies, the Rashba interaction is not effective and the transmissions are weak functions of energy, as discussed in Sec. IV. Then, the transport contribution to the Seebeck coefficient, $S \propto \partial \ln G / \partial E_{F}$, is negligible and $S$ tends to zero as $1 / E_{F}$. At low energies, the constant term exceeds the transport contribution due to the $k_{B} T_{0} / E_{F}$ term [60]. Therefore, the transport term is relevant only at intermediate energies, as shown in Fig. 7.

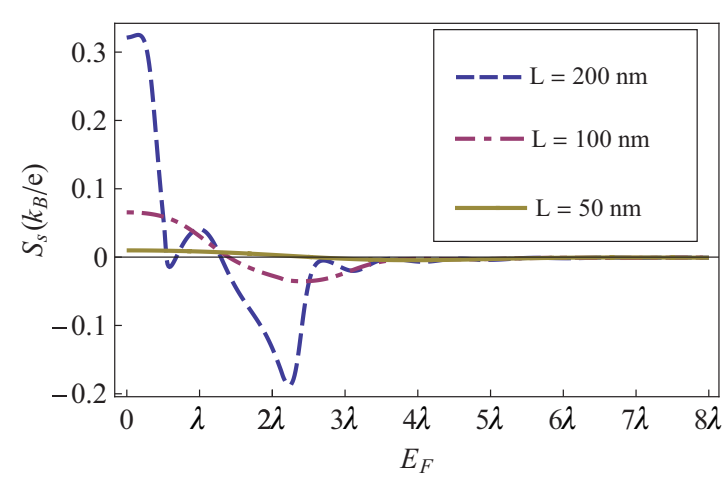

FIG. 8. (Color online) Subband-Seebeck coefficient as a function of Fermi energy for different values of the spin-orbit region length. Parameters: $\lambda=10 \mathrm{meV}$ and $T_{0}=1 \mathrm{~K}$.

Applied temperature gradients can also lead to spin accumulations in the attached leads, as recently demonstrated in systems driven by spin Seebeck effects [49,50]. Then, it is natural to ask whether a local spin-orbit interaction in graphene leads to different subband populations. We address this question by calculating from Eqs. (23) and (24) the subband bias $V_{s}$ generated when $I=0$ and $I_{s}=0$ but $\Delta T \neq 0$. The subband thermopower $S_{s}=V_{s} / \Delta T$ then follows,

$$
S_{s}=-\left(\frac{\mathcal{L}_{+}}{G_{+}}-\frac{\mathcal{L}_{-}}{G_{-}}\right) .
$$

Notice that to obtain this result, we need to apply a bias voltage:

$$
V=-\frac{1}{2}\left(\frac{\mathcal{L}_{+}}{G_{+}}+\frac{\mathcal{L}_{-}}{G_{-}}\right) \Delta T .
$$

At low temperature, we can substitute Eq. (26) in Eq. (30), yielding

$$
S_{s}=-\frac{\pi^{2} k_{B}^{2} T_{0}}{3 e}\left(\frac{\partial \mathcal{T}_{+} / \partial E_{F}}{\mathcal{T}_{+}}-\frac{\partial \mathcal{T}_{-} / \partial E_{F}}{\mathcal{T}_{-}}\right) .
$$

Notably, the intrinsic thermopower of Eq. (29) drops out from the subband Seebeck coefficient in Eq. (32). $S_{s}$ depends only on the transmission probabilities to cross the spin-orbit region and is thus a purely transport property. Therefore, we expect a stronger energy dependence of the subband thermopower as compared with its charge analog, given by Eq. (28). This is confirmed in our numerical simulations. In Fig. 8, we represent $S_{s}$ as a function of $E_{F}$. The energy variation of the subband thermopower becomes more pronounced for wider spin-orbit regions because the subband-resolved transmissions differ strongly as the region size enhances. In addition, we observe a sign change of $S_{s}$, implying that for a positive difference of temperatures, a positive or negative subband potential is generated depending on the Fermi energy. As expected, for high energies, electrons are insensitive to the Rashba scattering potential and the subband thermopower tends to zero.

\section{CONCLUSIONS}

We have investigated the electric and thermoelectric properties of a graphene monolayer with inhomogeneous Rashba spin-orbit interaction patterned as a stripe along the sample. 
We have discussed the energy splittings due to the Rashba coupling and their effect in the transmission probabilities. Importantly, the existence of a critical angle for only one of the two subband states leads to a finite polarization when the externally modulated Fermi energy is of the order of the spin-orbit strength.

We have found that our system is more sensitive to temperature shifts than to potential differences. Surprisingly enough, the thermopower is dominated by an intrinsic term which is independent of the scattering potential. The strong energy variation is recovered when the thermopower is calculated in the subband space. Then, an applied temperature bias creates a subband polarization, which can attain significant values (positive or negative) at low Fermi energies.

We have considered a system free of disorder or scattering centers additional to the spin-orbit coupling. In a realistic sample, diffusion processes should be taken into account. However, it is remarkable that in the diffusive regime, a similar intrinsic thermopower $\left(S \sim k_{B}^{2} T_{0} / e E_{F}\right)$ is obtained [45]. Therefore, further work is needed to clarify the behavior of the Seebeck coefficient in the transition from the diffusive regime to the ballistic (quantum) regime considered here. Another interesting route would focus on the role of phonons
[61]. However, we do not expect that our results will change qualitatively since the phonon contribution is negligible at the low temperatures considered in our work.

Our results might be tested in a suspended graphene sample with a central section deposited onto a metallic substrate, inducing a spin-orbit interaction. The coupling between the monolayer and the metal can be tuned with an external electric field. Then, thermovoltages and thermocurrents would be detected upon local heating of a sample side. An alternative measurement would consider heating currents generated in response to an applied electric current under vanishing thermal gradients (Peltier effect). Due to reciprocity, the measured response can be related to the thermopower. Finally, hot electrons can originate from sample irradiation, as recently demonstrated in Refs. [62,63]. Our results are thus relevant for the exciting area that emphasizes the interplay between spin interactions and thermoelectric effects in graphene and related nanostructures.

\section{ACKNOWLEDGMENT}

This work has been supported by MINECO under Grant No. FIS2011-23526.
[1] K. S. Novoselov, A. K. Geim, S. V. Morozov, D. Jiang, Y. Zhang, S. V. Dubonos, I. V. Grigorieva, and A. A. Firsov, Science 306, 666 (2004)

[2] K. S. Novoselov, A. K. Geim, S. V. Morozov, D. Jiang, I. V. Grigorieva, S. V. Dubonos, and A. A. Firsov, Nature (London) 438, 197 (2005).

[3] A. H. Castro Neto, F. Guinea, N. M. R. Peres, K. S. Novoselov, and A. K. Geim, Rev. Mod. Phys. 81, 109 (2009).

[4] A. K. Geim and K. S. Novoselov, Nat. Mater. 6, 183 (2007).

[5] A. K. Geim, Science 324, 1530 (2009).

[6] C. W. J. Beenakker, Rev. Mod. Phys. 80, 1337 (2008).

[7] A. Varykhalov, J. Sanchez-Barriga, A. M. Shikin, C. Biswas, E. Vescovo, A. Rybkin, D. Marchenko, and O. Rader, Phys. Rev. Lett. 101, 157601 (2008).

[8] Yu. S. Dedkov, M. Fonin, U. Rüdiger, and C. Laubschat, Phys. Rev. Lett. 100, 107602 (2008).

[9] E. I. Rashba, Phys. Rev. B 79, 161409(R) (2009).

[10] C. Ertler, S. Konschuh, M. Gmitra, and J. Fabian, Phys. Rev. B 80, 041405(R) (2009).

[11] S. Abdelouahed, A. Ernst, J. Henk, I. V. Maznichenko, and I. Mertig, Phys. Rev. B 82, 125424 (2010).

[12] Z. Y. Li, Z. Q. Yang, S. Qiao, J. Hu, and R. Q. Wu, J. Phys.: Condens. Matter 23, 225502 (2011)

[13] E. I. Rashba, Fiz. Tverd. Tela (Leningrad) 2, 1224 (1960) [Sov. Phys. Solid State 2, 1109 (1960)].

[14] Y. Bychkov and E. I. Rashba, J. Phys. C 17, 6039 (1984).

[15] C. L. Kane and E. J. Mele, Phys. Rev. Lett. 95, 226801 (2005).

[16] C. L. Kane and E. J. Mele, Phys. Rev. Lett. 95, 146802 (2005).

[17] J. Nitta, T. Akazaki, H. Takayanagi, and T. Enoki, Phys. Rev. Lett. 78, 1335 (1997).

[18] A. V. Moroz and C. H. W. Barnes, Phys. Rev. B 60, 14272 (1999).

[19] F. Mireles and G. Kirczenow, Phys. Rev. B 64, 024426 (2001).

[20] D. Sánchez and Ll. Serra, Phys. Rev. B 74, 153313 (2006).
[21] J.-S. Jeong and H.-W. Lee, Phys. Rev. B 74, 195311 (2006).

[22] L. Zhang, F. Zhai, and H. Q. Xu, Phys. Rev. B 74, 195332 (2006).

[23] C. A. Perroni, D. Bercioux, V. Marigliano Ramaglia, and V. Cataudella, J. Phys.: Condens. Matter 19, 186227 (2007).

[24] K. Shen and M. W. Wu, Phys. Rev. B 77, 193305 (2008).

[25] D. Sánchez, L1. Serra, and M.-S. Choi, Phys. Rev. B 77, 035315 (2008).

[26] J. C. Egues, G. Burkard, and D. Loss, Phys. Rev. Lett. 89, 176401 (2002).

[27] P. San-José and E. Prada, Phys. Rev. B 74, 045305 (2006).

[28] F. Mazza, B. Braunecker, P. Recher, and A. Levy Yeyati, Phys. Rev. B 88, 195403 (2013).

[29] S. M. Frolov, S. Lüscher, W. Yu, Y. Ren, J. A. Folk, and W. Wegscheider, Nature (London) 458, 868 (2009).

[30] A. F. Sadreev and E. Ya. Sherman, Phys. Rev. B 88, 115302 (2013).

[31] M. O. Hachiya, G. Usaj, and J. C. Egues [Phys. Rev. B (to be published)], arXiv:1310.3707.

[32] T. Matsuyama, C.-M. Hu, D. Grundler, G. Meier, and U. Merkt, Phys. Rev. B 65, 155322 (2002).

[33] M. Khodas, A. Shekhter, and A. M. Finkelstein, Phys. Rev. Lett. 92, 086602 (2004).

[34] M. G. Pala, M. Governale, J. König, and U. Zülicke, Europhys. Lett. 65, 850 (2004).

[35] O. Entin-Wohlman, A. Aharony, Y. Tokura, and Y. Avishai, Phys. Rev. B 81, 075439 (2010).

[36] M. M. Gelabert and Ll. Serra, Eur. Phys. J. B 79, 341 (2011).

[37] A. Yamakage, K. I. Imura, J. Cayssol, and Y. Kuramoto, Europhys. Lett. 87, 47005 (2009).

[38] M. Rataj and J. Barnaś, Appl. Phys. Lett. 99, 162107 (2011).

[39] T. Stauber and J. Schliemann, New J. Phys. 11, 115003 (2009).

[40] Kh. Shakouri, M. R. Masir, A. Jellal, E. B. Choubabi, and F. M. Peeters, Phys. Rev. B 88, 115408 (2013).

[41] D. Bercioux and A. De Martino, Phys. Rev. B 81, 165410 (2010). 
[42] M. H. Liu, J. Bundesmann, and K. Richter, Phys. Rev. B 85, 085406 (2012).

[43] N. W. Ashcroft and N. D. Mermin, in Solid State Physics (Saunders College, Philadelphia, PA, 1976), pp. 253-258.

[44] D. Dragoman and M. Dragoman, Appl. Phys. Lett. 91, 203116 (2007).

[45] Y. M. Zuev, W. Chang, and P. Kim, Phys. Rev. Lett. 102, 096807 (2009).

[46] P. Wei, W. Bao, Y. Pu, C. N. Lau, and J. Shi, Phys. Rev. Lett. 102, 166808 (2009).

[47] O. Shevtsov, P. Carmier, C. Groth, X. Waintal, and D. Carpentier, Phys. Rev. B 85, 245441 (2012).

[48] G. E. W. Bauer, A. H. MacDonald, and S. Maekawa, Solid State Commun. 150, 459 (2010).

[49] K. Uchida, S. Takahashi, K. Harii, J. Ieda, W. Koshibae, K. Ando, S. Maekawa, and E. Saitoh, Nature (London) 455, 778 (2008).

[50] C. M. Jaworski, J. Yang, S. Mack, D. D. Awschalom, J. P. Heremans, and R. C. Myers, Nat. Mater. 9, 898 (2010).

[51] D. Huertas-Hernando, F. Guinea, and A. Brataas, Phys. Rev. B 74, 155426 (2006).

[52] H. Min, J. E. Hill, N. A. Sinitsyn, B. R. Sahu, L. Kleinman, and A. H. MacDonald, Phys. Rev. B 74, 165310 (2006).
[53] Ll. Serra, D. Sánchez, and R. López, Phys. Rev. B 76, 045339 (2007).

[54] M. I. Katsnelson, K. S. Novoselov, and A. K. Geim, Nat. Phys. 2, 620 (2006).

[55] C. W. J. Beenakker and H. van Houten, Solid State Phys. 44, 1 (1991).

[56] T. Rejec, R. Žitko, J. Mravlje, and A. Ramšak, Phys. Rev. B 85, 085117 (2012).

[57] J. S. Lim, R. López, and D. Sánchez, New J. Phys. 16, 015003 (2014).

[58] A. S. Dzurak, C. G. Smith, C. H. W. Barnes, M. Pepper, L. Martín-Moreno, C. T. Liang, D. A. Ritchie, and G. A. C. Jones, Phys. Rev. B 55, R10197 (1997).

[59] P. Streda, J. Phys.: Condens. Matter 1, 1025 (1989).

[60] The $1 / E_{F}$ divergence at very low energies below $k_{B} T_{0}$ is just an artifact of our Sommerfeld expansion, which is exact up to $\left(k_{B} T_{0} / E_{F}\right)^{4}$. Near the charge neutrality point, $E_{F}$ is of the order of $K_{B} T_{0}$ and the Sommerfeld expansion breaks down.

[61] E. Munoz, J. Phys. Condens. Matter 24, 195302 (2012).

[62] N. M. Gabor, J. C. W. Song, Q. Ma, N. L. Nair, T. Taychatanapat, K. Watanabe, T. Taniguchi, L. S. Levitov, and P. Jarillo-Herrero, Science 334, 648 (2011).

[63] M. Freitag, T. Low, and P. Avouris, Nano Lett. 13, 1644 (2013). 